\title{
Performance of SAC OCDMA-FSO communication systems
}

\begin{abstract}
In this paper, the performance of free space optical (FSO) communication system employing the spectral amplitude coding optical code division multiple access (SAC OCDMA) technique is presented. SAC OCDMA is one of the multiplexing schemes that have become a research area of interest in optical communication because of its flexibility in allocating channels, ability to operate asynchronously, enhanced privacy and increased network capacity. It utilizes Khazaniï Syed (KS) code with spectral direct decoding (SDD) technique. The SAC OCDMA-FSO communication system was compared with the FSO system employing intensity modulation/direct detection (IM/DD) technique. The results of this study show that the performance of the proposed system is better than the system employing the IM/DD technique.
\end{abstract}

Keyword: Free space optics (FSO); SAC OCDMA; Khazaniï Syed (KS) code; Spectral direct decoding (SDD) 